\title{
Collecte, tri et caractérisation des accessions de maïs (Zea mays L.) de décrue cultivées au niveau de la haute et moyenne vallée du fleuve Sénégal (cas de la Mauritanie)
}

Ibrahima Cheikhou TRAORÈ ${ }^{1}$, Nianguiri Moussa KONATE ${ }^{2}$, Elhadji FAYE ${ }^{3}$, Habibou Mbaye GUEYE ${ }^{4}$ et Hamidou DIENG ${ }^{1}$ *

${ }^{1}$ Département de Biologie, Faculté des Sciences et Techniques, B.P. 5026, Université de Nouakchott Al Aasriya (UNA), Nouakchott, Mauritanie.

2Département de Production et de Protection Végétale, Institut Supérieur de l'Enseignement Technologique (ISET), Unité de Recherche Ressources Génétiques et Environnement, Rosso, Mauritanie.

${ }^{3}$ Institut Supérieur de Formation Agricole et Rurale (ISFAR) ex ENCR de BAMBEY. B.P. 54 Bambey, Sénégal.

${ }^{4}$ CentreNational de Recherche Agronomique et du Développement Agricole (CNRADA) B.P. 22 Kaédi, Mauritanie.

*Auteur correspondant, dihamidon@yaboo.fr, Tel:00 2224 6443347/0022222228244.

Publication date 31/12/2019, http://www.m.elewa.org/JAPS

1 RÉSUMÉ :

L'objectif de cette étude était de collecter, trier et étudier la diversité phénotypique des accessions de maïs de décrue sur la base de quelques variables sélectionnées, parmi les descripteurs du maïs. Cent soixante-deux (162) accessions de maïs de décrue collectées dans la haute et moyenne vallée du fleuve Sénégal ont été ainsi triées et évaluées au plan morphologique. Parmi ces 162 accessions, 64,81\% sont collectées au niveau de la haute vallée et 35,19 \% dans la moyenne vallée. En se basant sur trois caractères morphologiques du maïs, (couleurs des grains, texture des grains et couleur de la rafle), le tri de cette collection a montré une très grande variabilité en morphotypes (15), avec la dominance du morphotype Jaune-Corné-Blanc (J-C-B) dans la collection avec un effectif (85/162). Les morphotypes avérés très rares ne dépassent pas l'effectif 1 dans la collection. La caractérisation sur épis de ces morphotypes en fonction de certains caractères (poids de l'épi et le poids de 100 graines, longueur de l'épi,...), liés au rendement a montré une diversité phénotypique très importante. En effet, le poids de l'épi varie de $63,03 \mathrm{~g}$ pour les morphotypes à petits épis à $290 \mathrm{~g}$ pour les morphotypes à gros épis de même que pour la longueur de l'épi qui varie de $10,5 \mathrm{~cm}$ à $26 \mathrm{~cm}$. Cette diversité offre une grande possibilité de choix des géniteurs pour la création de variétés améliorées de maïs ayant un potentiel de rendement élevé et adaptées à différentes zones agro-écologiques de la vallée du fleuve Sénégal.

\section{ABSTRACT}

The objective of this study was to collect, sort and study the phenotypic diversity of flood recession maize accessions based on a few selected variables, among the maize descriptors. One hundred and sixty-two (162) flood recession maize accessions collected in the high and medium valley of the Senegal River were thus sorted and evaluated morphologically.Among these 162 accessions, $64.81 \%$ are collected in the upper valley and $35.18 \%$ in the middle valley. Based on three morphological characteristics of the maize (grain colors, texture and 
color of the stalk), the sorting of this collection showed a great variability in morphotypes (15), with the dominance of the morphotype Yellow-Corné-Blanc (JCB) in the collection with a strength (85/162). The morphotypes found to bevery rare do not exceed the number 1 in the collection. Characterization on the ears of these morphotypes according to some characteristics (weight of the ear and the weight of $100 \mathrm{~g}$, length of the ear), related to the yield showed a very important phenotypic diversity. In fact, the weight of the ear varies from $63.03 \mathrm{~g}$ for short-eared morphotypes to $290 \mathrm{~g}$ for coarse-cut morphotypes as well as for spike length ranging from $10.5 \mathrm{~cm}$ to $26 \mathrm{~cm}$. This diversity offers a great opportunity for brood stock selection for the creation of improved maize varieties with high yield potential and adapted to different agro-ecological zones of the Senegal River Valley.

\section{INTRODUCTION}

Le maïs (Zea mays L.) est une graminée céréalière importante pour beaucoup de pays, surtout pour sa production mondiale 839 millions de tonnes en 2013 contre 653 millions pour le blé (Kahndo et al., 2015). En Afrique de l'ouest et du centre, le maïs représente un peu plus de $20 \%$ de la production alimentaire (Rouanet, 1984) et sa production est estimée à 18,5 million de tonnes (Boua et al., 2016). En Mauritanie, le maïs a longtemps occupé une place de choix parmi d'autres céréales, bien que sa production nationale reste encore faible (2979 tonnes pour une superficie 3943 ha, soit 0,75 t.ha ${ }^{-1}$ (DSIA/MDR, 2018). La culture du maïs, surtout de décrue, occupe une part importante de l'activité agricole des agriculteurs de la rive droite du fleuve Sénégal. Cette production est utilisée sous différentes formes : maïs grillé, couscous, bouillies de maïs et aussi comme fourrage pour le bétail.

Pour faire face à l'instabilité du climat, surtout la rareté et la fluctuation des pluies le plus souvent déficitaires, les producteurs de la rive droite du fleuve Sénégal, utilisent plusieurs techniques culturales traditionnelles, transmises de génération en génération axées surtout sur l'utilisation des écotypes locaux pour garantir une production certes faible, mais sécurisée.

Il existe ainsi, plusieurs formes traditionnelles locales de maïs de décrue, mal connues des sélectionneurs modernes, mais jalousement conservées par les producteurs. Selon Missihoun et al. (2012), les variétés locales représentent l'essentiel du matériel végétal utilisé par les producteurs et constituent la matière première devant être utilisée par les sélectionneurs pour améliorer la productivité et la qualité nutritionnelle. Elles présentent en plus une meilleure adaptation aux conditions climatiques et pédologiques de leur région d'origine (Hammer et Teklu, 2008). La connaissance des meilleurs écotypes de ce matériel, pourra à la suite de son utilisation, contribuer à l'autosuffisance alimentaire et à la sécurité nutritionnelle des populations. Malgré les efforts de la communauté scientifique mauritanienne, très peu de données scientifiques sont disponibles sur ces importantes ressources phytogénétiques de maïs de décrue. Il est donc nécessaire, voire indispensable de conduire des recherches sur la nature, les performances et la diversité du maïs de décrue, un patrimoine génétique à identifier et à sauvegarder. Pour une meilleure connaissance de l'existant, quatre étapes sont nécessaires :

- L'organisation des prospections de collecte de ces écotypes au niveau des différentes zones agro-écologiques de la vallée. Cette collecte est le point de départ pour déceler et localiser les meilleurs écotypes (Marchay et Lagarde, 1987) et peut en assurer une meilleure conservation (Labeyrie et al., 2014);

- $\quad$ la rencontre avec les producteurs utilisateurs pour une collecte d'informations surtout, en ce qui concerne les techniques culturales et les connaissances traditionnelles sur les écotypes transmis de génération en génération; 
- l'analyse de la diversité génétique par le tri et la caractérisation des accessions collectées sur la base des méthodes morphologiques et biologiques ;

- $\quad$ la formulation de recommandations aux producteurs utilisateurs des écotypes pour la production mais aussi pour les sélectionneurs.

\section{MATÉRIEL ET MÉTHODES}

3.1 Zone d'étude et sites de collectes : La collecte des accessions de maïs de décrue a eu lieu entre mars et avril 2018 dans deux zones agro-écologiques de la rive droite du fleuve Sénégal; haute et moyenne vallée (Figure 1). Dans chacune de ces deux zones agro-
L'objectif de la présente étude est de contribuer à une meilleure connaissance et à la valorisation de la performance des écotypes de maïs de décrues collectées dans la haute et moyenne vallée de la rive droite du fleuve Sénégal.

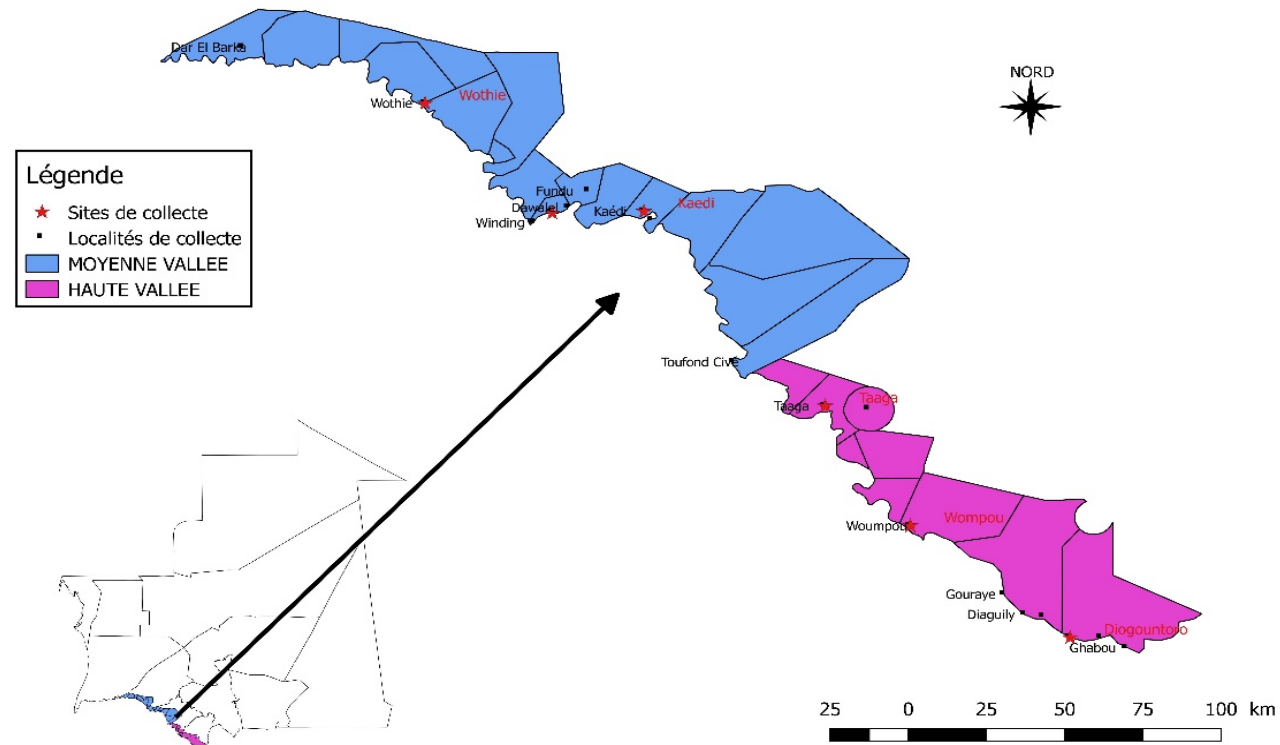

Carte de situation des zones agroécologiques de la rive droite du fleuve Sénégal réalisée par Oumar DIAGANA à partir des données du terrain à l'aide du logiciel QGIS.

Figure 1: Localités et sites de collecte des accessions de maïs de décrue au niveau de la haute et moyenne vallée du fleuve Sénégal (collecte réalisée en 2018).

\subsection{Technique de collecte et mode de} prélèvement des accessions : Dans chacune des localités visitées, les accessions ont été collectées sur la base des observations directes, à la suite des entretiens avec les producteurs, qui détiennent des informations sur la nature, les performances et les origines des accessions. Cette méthode est le plus souvent utilisée lors des prospections et de la collecte pour plusieurs écologiques, la collecte est faite sur trois sites distants d'au moins $50 \mathrm{~km}$ (en rouge sur la figure 1). Pour chacun de ces trois sites, trois localités sont ciblées comme points de collecte au centre, en amont et en aval parallèlement au fleuve Sénégal (en noir sur la figure 1). 
3.3.1 Tri des accessions collectées : Nous avons utilisé trois caractères sélectionnés parmi les descripteurs du maïs (IPGRI, 1991) pour le tri des accessions collectées. Il s'agit de la couleur de la rafle, de la couleur et de la texture des grains. La combinaison de ces trois caractères nous a permis de regrouper les accessions collectées en morphotypes existants dans la rive droite du fleuve Sénégal (Tableau 1).

Tableau 1 : Principaux caractères utilisés pour le tri des accessions collectées

\begin{tabular}{c|c|c}
\hline Descripteurs & Possibilité & Remarque \\
\hline Couleur du grain & $\begin{array}{c}\text { Jaune ; blanc ; violet clair ; violet } \\
\text { foncé ; rouge ; bigarré ; brun ; } \\
\text { tacheté }\end{array}$ & $\begin{array}{c}\text { Choix de la couleur dominante sur } \\
\text { l'épi }\end{array}$ \\
\hline Texture des grains & $\begin{array}{c}\text { Denté ; semi-denté, intermédiaire } \\
\text { entre denté et corné, mais plus } \\
\text { proche de denté ; semi-corné, } \\
\text { corné avec extrémité molle ; corné } \\
;\end{array}$ & $\begin{array}{c}\text { Choix de la texture dominante sur } \\
\text { l'épi }\end{array}$ \\
\hline Couleur de la rafle & $\begin{array}{c}\text { Blanc ; rouge ; brun ; pourpre ; } \\
\text { bigarré ; violet }\end{array}$ & $\begin{array}{c}\text { Généralement la couleur de la rafle } \\
\text { est unique }\end{array}$ \\
\hline
\end{tabular}

Source adapté d'IPGRI (1991)

\subsubsection{Caractérisation des morphotypes} obtenus : Pour étudier la diversité génétique des accessions de maïs de décrue utilisées au niveau de la rive droite, nous avons procédé à la caractérisation de tous les morphotypes obtenus en utilisant cinq caractères quantitatifs liés à la productivité de maïs (Tshibingu et al., 2017). Il s'agit du poids de l'épi sans spathe (POE), du poids des cent grains (P100G), de la longueur de l'épi (LOE), du nombre de rangées par épi et du diamètre de l'épi (DIE).

\section{RESULTATS}

4.1 Bilan de collecte: Dix-huit localités ont été visitées entre mars et avril 2018 dans les différents sites de la haute et de la moyenne vallée du fleuve Sénégal. Au total 162 accessions ont été colletées dont $64,81 \%$ dans
3.4 Analyse statistique des données : Les données de collecte, de tri et de caractérisation morphologique ont été soumises à une analyse descriptive (fréquence, moyenne, pourcentage) à l'aide du logiciel SPSS 22.0. Les résultats sont présentés sous forme de tableaux et de graphiques construits avec le logiciel Excel. La comparaison des moyennes par le test de la plus petite différence significative (LSD) a été faite à l'aide du logiciel STATISTIX 10.0.

la haute vallée et 35,18 \% dans la moyenne vallée (Tableau 2). Dans certaines localités de la moyenne vallée nous n'avons pas trouvé d'accessions en raison d'une mauvaise récolte de l'année précédente.

Tableau 2 : Nombre d'accessions obtenues en fonction des localités et des zones agro-écologiques

\begin{tabular}{c|c|c|c}
\hline Zones agro-écologiques & $\begin{array}{c}\text { Nombre de } \\
\text { localités }\end{array}$ & $\begin{array}{c}\text { Nombre } \\
\text { d'accessions } \\
\text { collectées }\end{array}$ & $\begin{array}{c}\text { \% des accessions } \\
\text { collectées }\end{array}$ \\
\hline Haute vallée & 09 & 105 & 64,81 \\
\hline Moyenne vallée & 09 & 57 & 35,18 \\
\hline Total & 18 & 162 & 100 \\
\hline
\end{tabular}


4.2 Bilan du tri de la collection des accessions : La combinaison des trois caractères descripteurs (couleur de la graine, texture des grains et la couleur de la rafle) a permis de regrouper les 162 accessions en 15 morphotypes dont 11 morphotypes dans la haute vallée et 9 dans la moyenne vallée (Tableau 3). Quelques morphotypes sont retrouvés également dans la haute vallée et dans la moyenne vallée. Ainsi les morphotypes jaune-corné-blanche dominent dans la collection avec un effectif de (64/105) dans la haute vallée et $(21 / 57)$ dans la moyenne vallée (Figure 2). Les morphotypes avérés très rares ne dépassent pas l'effectif 1 dans la collection.

Tableau 3 : Nombre de morphotypes en fonction des accessions collectées

\begin{tabular}{c|c|c}
\hline Zone agro-écologique & Nombre d'accessions & $\begin{array}{c}\text { Nombre de morphotypes } \\
\text { obtenus après le tri }\end{array}$ \\
\hline Haute vallée & 105 & 11 \\
\hline Moyenne vallée & 57 & 09 \\
\hline Total & 162 & $* * *$ \\
\hline
\end{tabular}

*** : La somme est impossible dans le cas où les mêmes morphotypes peuvent se retrouver aussi bien en haute et moyenne vallée

Tableau 4: Exemples de morphotypes regroupés, en se basant sur la couleur de l'épi, la texture des graines et sur la couleur de la rafle (un épi peut avoir la même couleur des grains mais des couleurs de la rafle différentes).

\begin{tabular}{|c|c|c|c|}
\hline Morphotypes & Couleur de l'épi & Texture des grains & Couleur de la rafle \\
\hline $\mathrm{J}-\mathrm{C}-\mathrm{B}$ & 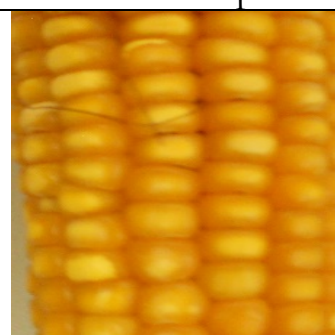 & 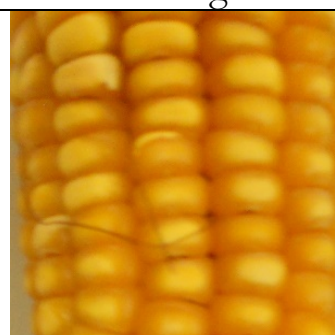 & 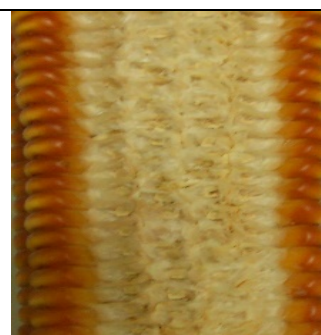 \\
\hline & Jaune & Corné & Blanche \\
\hline B-D-R & Blanc & Denté & 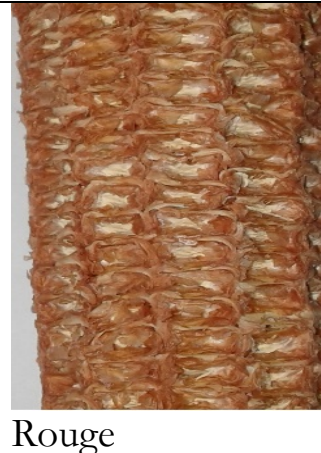 \\
\hline
\end{tabular}




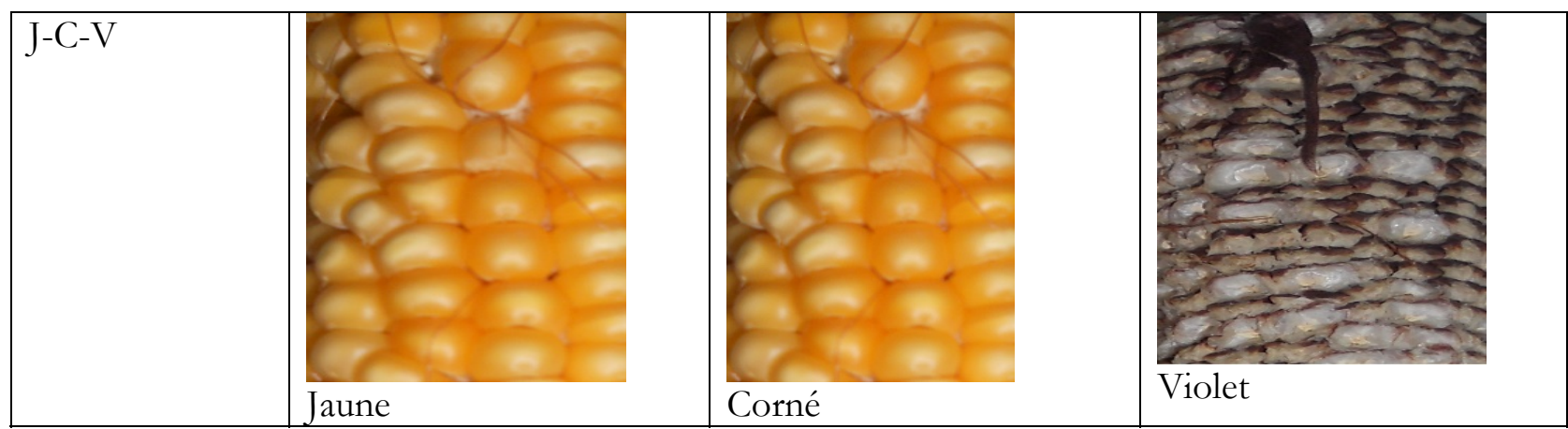

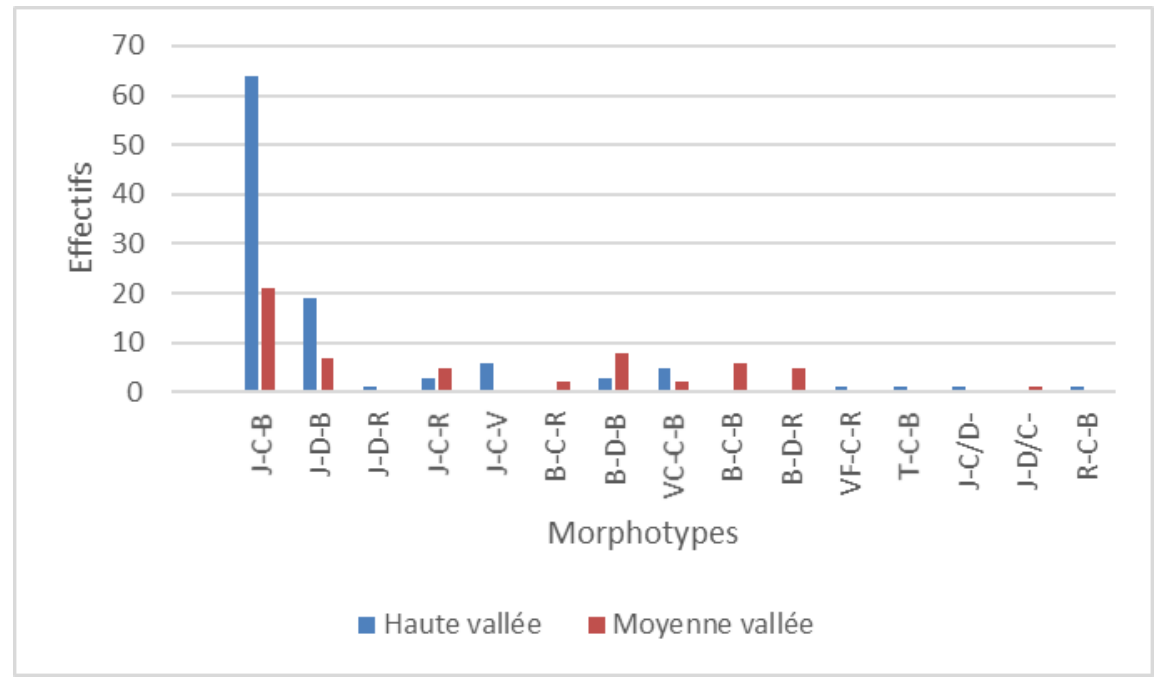

Figure 2 : Effectifs d'apparition des morphotypes au niveau de la collection et en fonction des zones agro-écologiques

4.3 Analyse de la pertinence des différents morphotypes en fonction des sites: Une variabilité très importante des morphotypes en fonction des sites a été observée dans la zone d'étude. Le plus fort taux de variabilité de morphotype (09) a été observé dans le site de M'Bagne, et le plus faible (03) dans le site de Wothie (Figure 3). 


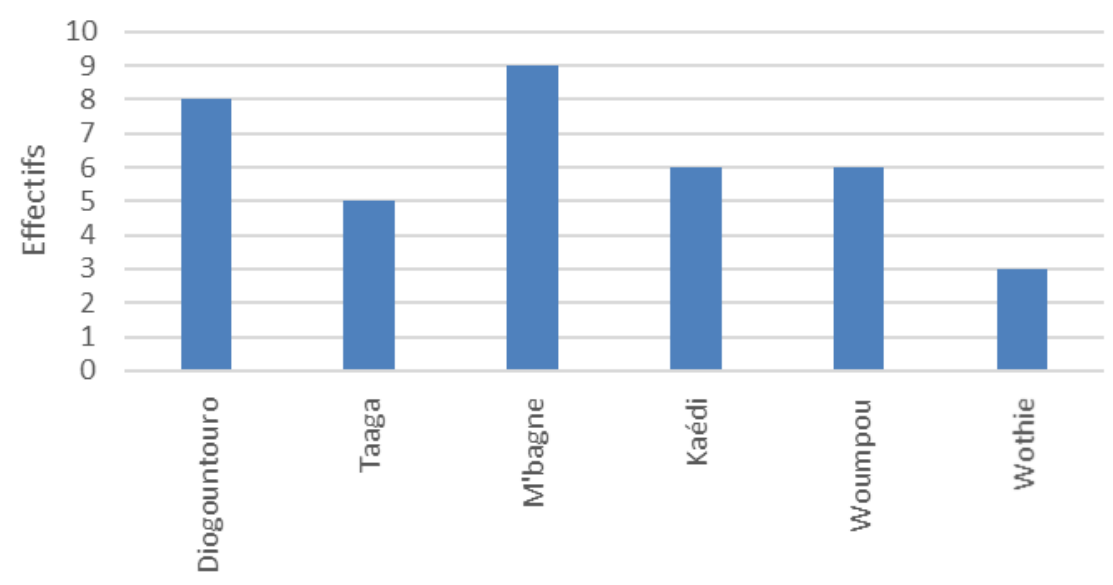

Morphotypes observés en fonction des sites

Figure 3 : Nombre de morphotypes observés en fonction des sites

4.4 Caractérisation morphologique sur épis des morphotypes : Les paramètres de production (Tableau 4) ont été mesurés sur l'ensemble des morphotypes obtenus. Des écarts importants ont été observés entre les valeurs minimales et maximales pour l'ensemble des caractères. Par exemple, le poids de l'épi sans spathe varie de 63,03 g pour les morphotypes à petit épis à $290 \mathrm{~g}$ pour les morphotypes à gros épis, de même pour la longueur de l'épi qui varie de $10,5 \mathrm{~cm}$ à $26 \mathrm{~cm}$. En moyenne, les morphotypes ont une taille de $18,16 \mathrm{~cm}$ et un poids de $170,01 \mathrm{~g}$.

Tableau 4 : Valeur minimale, maximale, moyenne et écart-type de 5 caractères quantitatifs étudiés

\begin{tabular}{llllll}
\hline Variable & $\begin{array}{l}\text { Nombre de } \\
\text { morphotypes } \\
\text { observés }\end{array}$ & $\begin{array}{l}\text { Valeur } \\
\text { minimale }\end{array}$ & $\begin{array}{l}\text { Valeur } \\
\text { maximale }\end{array}$ & Moyenne & $\begin{array}{l}\text { Ecart- } \\
\text { type }\end{array}$ \\
\hline Longueur de l'épi $(\mathrm{cm})$ & 15 & 10,50 & 26,00 & 18,16 & 2,80 \\
\hline Diamètre de l'épi $(\mathrm{cm})$ & 15 & 6,00 & 20,50 & 13,76 & 1,70 \\
\hline Nombre de rangée /épi & 15 & 10,00 & 22,00 & 15,09 & 2,40 \\
\hline $\begin{array}{l}\text { Poids de l'épi sans } \\
\text { spathe (g) }\end{array}$ & 15 & 63,03 & 290,00 & 170,01 & 46,53 \\
\hline Poids de 100 grains $(\mathrm{g})$ & 15 & 16,40 & 46,73 & 27,38 & 5,88 \\
\hline
\end{tabular}

4.5 Comparaison des performances des morphotypes : La comparaison des valeurs moyennes des paramètres de production de maïs par la plus petite différence significative (LSD) a été effectuée sur les morphotypes de la collection (Tableau 5). La longueur de l'épi, le nombre de rangées par épi, le poids de l'épi et le poids de 100 grains présentent des différences significatives entre les différents morphotypes. Les épis les plus courts ont été observés sur les morphotypes J-D-B avec 15, $10 \mathrm{~cm}$ et les plus longs ont été observés dans les morphotypes J-C-R avec $20,060 \mathrm{~cm}$. Le poids de l'épi et le poids de 100 grains le plus élevé a été observé avec les morphotypes B-DB et les plus petit avec les morphotypes J-D-B. Quant au nombre de rangées/épi les morphotypes J-D-B présentent le meilleur nombre de rangées/épi. 
Tableau 5 : Variabilité des morphotypes en fonction des paramètres de production

\begin{tabular}{l|l|l|l|l|l}
\hline Morphotypes & $\begin{array}{l}\text { Longueur épi } \\
(\mathrm{cm})\end{array}$ & $\begin{array}{l}\text { Diamètre épi } \\
(\mathrm{cm})\end{array}$ & $\begin{array}{l}\text { Nombre } \\
\text { rangée/épi }\end{array}$ & $\begin{array}{l}\text { Poids de } \\
\text { l'épi (gr) }\end{array}$ & $\begin{array}{l}\text { Poids } 100 \\
\text { grains } \\
(\mathrm{gr})\end{array}$ \\
\hline B-D-R & $\mathbf{1 8 , 7 2 0 a b}$ & $14,860 \mathrm{a}$ & $15,200 \mathrm{ab}$ & $\mathbf{2 0 9 , 1 5 a}$ & $\mathbf{3 2 , 1 9 8 a}$ \\
\hline B-D-B & $16,340 \mathrm{bc}$ & $14,480 \mathrm{a}$ & $13,600 \mathrm{ab}$ & $176,21 \mathrm{ab}$ & $30,092 \mathrm{ab}$ \\
\hline B-C-B & $15,260 \mathrm{c}$ & $14,360 \mathrm{a}$ & $14,000 \mathrm{ab}$ & $156,84 \mathrm{ab}$ & $28,696 \mathrm{abc}$ \\
\hline J-C-R & $\mathbf{2 0 , 0 6 0 a}$ & $13,640 \mathrm{a}$ & $13,200 \mathrm{~b}$ & $\mathbf{1 8 3 , 3 5 a b}$ & $\mathbf{3 1 , 3 2 2 a}$ \\
\hline VC-C-B & $17,960 \mathrm{abc}$ & $13,680 \mathrm{a}$ & $14,800 \mathrm{ab}$ & $164,74 \mathrm{ab}$ & $28,678 \mathrm{abc}$ \\
\hline J-C-V & $\mathbf{1 9 , 1 4 0 a b}$ & $12,900 \mathrm{a}$ & $15,200 \mathrm{ab}$ & $151,82 \mathrm{ab}$ & $29,850 \mathrm{ab}$ \\
\hline J-D-B & $15,100 \mathrm{c}$ & $12,900 \mathrm{a}$ & $\mathbf{1 7 , 2 0 0 a}$ & $124,98 \mathrm{~b}$ & $21,256 \mathrm{c}$ \\
\hline J-C-B & $18,100 \mathrm{abc}$ & $10,200 \mathrm{~b}$ & $\mathbf{1 6 , 0 0 0 a b}$ & $156,21 \mathrm{ab}$ & $22,240 \mathrm{bc}$ \\
\hline $\begin{array}{l}\text { Moyenne } \\
\text { générale }\end{array}$ & $\mathbf{1 7 , 5 8 5}$ & $\mathbf{1 3 , 3 7 7}$ & $\mathbf{1 4 , 9 0 0}$ & $\mathbf{1 6 5 , 4 1 2}$ & $\mathbf{2 8 , 0 4 1}$ \\
\hline CV & 14,600 & 14,130 & 19,970 & 29,370 & 23,740 \\
\hline Lsd & 3,300 & 2,430 & 3,830 & 62,580 & 8,570 \\
\hline
\end{tabular}

\section{DISCUSSION}

La prospection et la collecte constituent les étapes les plus importantes pour la sauvegarde des ressources phytogénétiques selon Marchay et Lagarde (1987). Plusieurs études ont montré que d'importantes collections de plantes cultivées ont été conservées par les communautés locales avec leurs modes de cultures traditionnelles et pratiques agricoles (Plucknett et al., 1983 ; Altieri et Merrick, 1987; Pei et Xu, 1997 ; Li et al., 2011). Pour étudier ce patrimoine important conservé, nous avons réalisé des prospections dans les différentes zones agro écologiques de la vallée du fleuve Sénégal en vue de collecter les ressources génétiques du maïs de décrue. Cette collecte a concerné 6 sites regroupant 18 localités, dans lesquelles 162 accessions ont été collectées, $64,81 \%$ dans la haute vallée et 35,18\% dans la moyenne vallée. La présente étude a révélé l'existence d'une diversité dans la combinaison des trois caractères descripteurs (couleur du grain, texture des grains et la couleur de la rafle). Quinze morphotypes ont été observés dans l'ensemble des 162 accessions collectées dont 11 morphotypes dans la haute vallée et 9 dans la moyenne vallée. Ainsi, le plus fort de taux de variabilité de morphotype (09) a été observé dans les sites de Diogountouro et M'bagne, et le plus faible (03) a été observé dans le site de Wothie (Figure 3). La présence de ces multitudes de morphotypes témoigne de l'existence probable d'une grande variabilité génétique des variétés locales de maïs cultivées dans les sites de collecte. Le taux de morphotypes élevé au niveau de M'bagne et Diogountouro peut s'expliquer par leur forte intensité de pratique culturale en milieux de décrue, pluvial et irrigué tandis que à Wothie la 
pratique culturale est surtout limitée en milieux de décrue et irrigué. Le morphotype jaunecorné-blanc (J-C-B) est le plus abondant dans la collection avec 52,46\% d'apparitions, suivi par le morphotype jaune-denté-blanc (J-D-B) avec $16 \%$. Leur prépondérance pourrait s'expliquer d'une part par les habitudes alimentaires et d'autre part par les pratiques plus maitrisées de gestions des semences. Plusieurs auteurs ont montré que les pratiques de gestion paysanne des semences, notamment les échanges de semences (variétés) entre agriculteurs sont à l'origine d'une diversité importante entre les populations de plantes cultivées (Mckey et al., 2001 ; Barnaud et al., 2008 ; Delaunay et al., 2008 ; Missihoun et al., 2012).

La caractérisation sur épis de ces quinze (15) morphotypes a révélé des écarts importants entre les caractères agronomiques de

\section{CONCLUSION}

L'étude est menée dans le cadre de la conservation et de la valorisation des ressources phytogénétiques de la rive droite du fleuve Sénégal. Elle a permis de collecter 162 accessions de maïs de décrue, dont 64,81\% dans la haute vallée et 35,18\% dans la moyenne vallée. Il est à retenir que ces accessions présentent une large variabilité de morphotypes, basée sur la combinaison de trois caractères descripteurs (la couleur des grains, la texture des grains et la couleur de la rafle) et sur les paramètres de production. Cette variabilité des morphotypes varie en fonction de la gestion paysanne des semences, notamment les échanges de semences entre les producteurs dans la haute et la moyenne vallée du fleuve

\section{REMERCIEMENTS}

Les auteurs remercient l'Université de Nouakchott Al Aasriya pour l'appui financier de cette étude à travers le projet de recherche production, ce qui peut s'expliquer par une forte variabilité génétique des accessions collectées. Le poids de l'épi sans spathe varie de 63,03 g pour les morphotypes à petit épis à 290 $\mathrm{g}$ pour les morphotypes à gros épis, de même pour la longueur de l'épi qui varie de $10,5 \mathrm{~cm}$ à $26 \mathrm{~cm}$. En moyenne, les morphotypes ont une taille de 18,16 cm et un poids de 170,01 g. La caractérisation permet d'avoir une première idée de la variabilité du matériel collecté et constitue une des étapes importantes dans la description et la classification du germoplasme des plantes cultivées (Manzano et al., 2001 ; Yobi et al., 2002 ; Radhouane, 2004). Au regard des moyennes des différents morphotypes sous l'analyse de la différence significative, les morphotypes Blanc-denté-rouge et Jaune corné-rouge, présentent les paramètres les plus pertinents liés au rendement.

Sénégal. Le faible échange de semence et les contraintes abiotiques et biotiques menacent la disparition de la variabilité et la disponibilité des morphotypes. En terme de variation sur les paramètres de production, les morphotypes jaune-corné -rouge présentent les épis les plus longs, le poids de l'épi et le poids de 100 grains les plus élevés. Cette variabilité constitue un atout pour les travaux de sélection. Il est donc urgent d'analyser l'importance socioculturelle de cette culture de décrue, d'entreprendre des études phylogénétique, agro-morphologique et génétique afin de mettre en place un programme de préservation, de conservation et d'amélioration génétiques.

«Caractérisation agro-morphologique des accessions de maïs de décrue collectées dans la vallée du fleuve Sénégal ».

\section{RÉFÉRENCES BIBLIOGRAPHIQUES}

Adoukonou-Sagbadja H, Dansi A, Vodouhè R, Akpagana K, 2006. Indigenous knowledge and traditional conservation

of Fonio millet (Digitaria exilis Stapf, Digitaria iburua Stapf) in Togo, 
Biodiversity and Conservation, 15, 23792395.

AltieriMA, Merrick LC. 1987. In situ conservation of crop genetic resources through maintenance of traditional farming systems. Economic Botany 41:8696.

Barnaud A, Joly H., Mckey D.B., Deu M., Khasah C., Monné S., Garine E. 2008. Gestion des ressources génétiques du sorgho (Sorghum bicolor) chez les Duupa (Nord Cameroun), Cahiers Agricultures, 17, 2,178-182.

Baoua I.B, Amadou. L, Bakoye .O.N, O. Abdoulaye, Baributsa .D, Murdock L.L .2016. Maize quality in markets in four West African countries. Journal of Stored Products Research 69 (2016) 2630.

Delaunay S, R-P, Tescar, A, Oualbego, K. Vom-Brocke, J. Lançon, 2008. La culture du coton ne bouleverse pas les échanges traditionnels de semences de sorgho. Cabiers Agricultures, 17-189- 194.

DSIA/MDR 2018 .Direction des Statistiques et de l'Information Agricole, Ministère de l'Agriculture et du Développement Rural.

Hammer K, Teklu Y. 2008. Plant Genetic Resources: Selected Issues from Genetic Erosion to Genetic Engineering, Journal of Agriculture and Rural Development in the Tropics and Subtropics 109 (1), 15-50.

IPGRI. 1991. Descriptors for Maize. CIMMYT / IPGRI Mexico City, Mexique / Rome, Italy. 100 pp.

KahndoP D, Louise A, René A, Gnonpo J N ; Patrice L K. 2015. Évaluation morphologique et nutritionnelle de variétés locales et améliorées de maïs (Zea mays L.) produites en Côte d'Ivoire, Afrique SCIENCE 11(3)- 181 - 196.

Labeyrie, V., Rono, B., Leclerc, C., 2014. How social organization shapes crop diversity: an ecological anthropology approach among Tharaka farmers of
Mount Kenya. Agriculture and Human Values 31, 97-107. Doi: 10.1007/s10460-013-9451-9.

Li Y, Long C, Kato K, Yang C, Sato K, 2011. Indigenous knowledge and traditional conservation of hulless barley (Hordeum vulgare) germplasm resources in the Tibetan communities of Shangri-la, Yunnan, SW China. Genetic Resources and Crop Evolution 58:645-655.

Manzano A.R., Nodals R.A.A., Gutiérrez R.A.I.M., Mayor F.Z., Alfonso C.L. 2001. Morphological and isoenzyme variability of taro (Colocasia esculenta $\mathrm{L}$. Schott) germplasm in Cuba. Plant Genetic Resources Newsletter, 126, 31-40.

Marchay, Lagarde, 1987. A la recherche des variétés locales de plantes cultivées. Paris : France, Lavoisier.

McKeye D, Emperaie L, Elias M, Pinton F, Robert T, Desmoulière S, Rival L. 2001. Gestion locales et dynamiques régionales de la diversité variétale du manioc en Amozonie. Genet. Sel. Evol., $33: 465-490$.

Missihoun A.A., Agbangla C., AdoukonouSagbadja H., Ahanhanzo C et Vodouhè R, 2012. Gestion traditionnelle et statut des ressources génétiques du sorgho (Sorghum bicolor L. Moench) au NordOuest du Bénin, International Journal of Biological Chemical Sciences 6, 10031018.

Pei SJ, Xu JC, 1997. Biodiversity and sustainability in swidden agroecosystems: problems and opportunities. In: Pei SJ et al (eds) Biodiversity in Swidden agroecosystems in Xishuangbanna. Yunnan Education Press, Kunming, pp 173-177.

Plucknett DL, Smith NJH, Williams JT.1983. Crop germplasm conservation and developing countries. Science 220: 63-169.

Rouanet.G 1984 .Le maïs GI collection René Coste, Maisonneuve et Larose et ACCT Paris ,12.5 pages. 
Radhouane L. 2004.. Etude de la variabilité morpho-phénologique chez Pennisetum glaucum (L.) R. Br., Plant Genetic Resources Newsletter, 138, 18-22.

Tshibingu Remy Mukendi, Théodore Tshilumba Mukadi, Maurice Mpoyi2 B., Benjamin Mutamba Ntatangolo, Dominique Kabongo Musenge, Meschack Ilunga Tshibingu, Judith Ngoie Kazadi, Dieudonné Ngoyi Nyembo, Theodore Munyuli
Mushambani. 2017. Évaluation de la productivité du maïs (Zea mays L.) sous amendements organique et minéral dans la province de Lomami, République Démocratique du Congo, Journal of Applied Biosciences 109: 10571-10579.

Yobi A., Henchi B., Neffati M., Jendoubi R. 2002.Système de reproduction et variabilité morpho-phénologique chez Allium roseum, Plant Genetic Resources Newsletter, 127, 29-34. 